\title{
La educación emocional en niños del nivel preescolar: una revisión sistemática
}

Emotional education in preschool children: a systematic review

Educação emocional em crianças pré-escolares: uma revisão sistemática

\section{ARTÍCULO GENERAL}

\author{
María Leonor Peña Julca \\ marileonor31@yahoo.com \\ https://orcid.org/0000-0002-0161-4480 \\ Pontificia Universidad Católica del Perú, Lima - Perú
}

Recibido 18 de Enero 2021 | Arbitrado y aceptado 10 de Marzo 2021 | Publicado en 05 Junio 2021

\section{RESUMEN}

En estos tiempos de grandes cambios tanto a nivel científico y tecnológico y por ende en el campo educativo, es necesario generar espacios que permitan afrontar temas relacionados con la educación emocional dada su gran relevancia al permitir formar plenamente a los estudiantes desde edades tempranas con respecto al manejo de sus emociones y los ayuden a afrontar el futuro de manera asertiva y tolerante.

Por ello, a través de esta investigación se dan a conocer los resultados obtenidos de la revisión de artículos científicos relacionados a la educación emocional. Esta investigación tiene como objetivo describir la situación de la educación emocional en niños del nivel preescolar en diferentes contextos educativos. Para lo cual se tomó en cuenta un rango en el tiempo desde el año 2016 al 2020, observándose pocas investigaciones que tomen en cuenta estudios referentes a niños de este nivel de escolaridad.

Mediante este artículo, en un primer momento se presentan diferentes conceptos relacionados con la educación emocional y las variables consideradas para su análisis como conciencia emocional, inteligencia emocional, regulación de las emociones y competencias sociales y emocionales; a través de diferentes puntos de vista y aportes dados por especialistas en esta temática. Por otro lado, se hace una revisión de los programas educativos propuestos para la implementación de la educación emocional en las escuelas atendiendo a las características, necesidades e intereses de los niños de educación preescolar.

Palabras claves: Educación emocional, conciencia emocional, inteligencia emocional, regulación de las emociones, competencias sociales y emocionales, educación preescolar.

\section{ABSTRACT}

In these times of great changes both at the scientific and technological level and therefore in the educational field, it is necessary to create spaces that allow facing issues related to emotional education given its great relevance by allowing students to fully train from an early age with respect to manage their emotions and help them face the future in an assertive and tolerant way.

Therefore, through this research, the results obtained from the review of scientific articles related to emotional education are made known. This research aims to describe the situation of emotional education in preschool children in different educational contexts. For which a range in time from 2016 to 2020 was taken into account, observing few investigations that take into account studies regarding children of this level of education.

Through this article, at first, different concepts related to emotional education and the variables considered for analysis such as emotional awareness, emotional intelligence, regulation of emotions and social and emotional competences are presented; through different points of view and contributions given by specialists in this matter. On the other hand, a review is made of the educational programs proposed for the implementation of emotional education in schools, taking into account the characteristics, needs and interests of preschool children.

Keywords: Emotional education, emotional awareness, emotional intelligence, emotion regulation, social and emotional competencies, preschool education.

\section{RESUMO}

Nestes tempos de grandes mudanças tanto a nível científico e tecnológico e, portanto, no campo educacional, é necessária a criação de espaços que permitam enfrentar as questões relacionadas com a educação emocional dada a sua grande relevância ao permitir aos alunos uma formação plena desde a mais tenra idade com respeito. para gerir as suas emoções e ajudá-los a enfrentar o futuro de forma assertiva e tolerante.

Assim, por meio desta pesquisa, são divulgados os resultados obtidos na revisão de artigos científicos relacionados à educação emocional. Esta pesquisa tem como objetivo descrever a situação da educação emocional em crianças pré-escolares em diferentes contextos educacionais. Para o qual foi levado em consideração um intervalo de tempo de 2016 a 2020, observando-se poucas investigações que contemplam estudos envolvendo crianças nesse nível de ensino.

Através deste artigo, num primeiro momento, são apresentados diferentes conceitos relacionados à educação emocional e às variáveis consideradas para análise como consciência emocional, inteligência emocional, regulação das emoções e competências sociais e emocionais; através de diferentes pontos de vista e contribuições de especialistas no assunto. Por outro lado, é feita uma revisão dos programas educacionais propostos para a implementação da educação emocional nas escolas, levando em consideração as características, necessidades e interesses dos pré-escolares.

Palavras-chave: Educação emocional, consciência emocional, inteligência emocional, regulação da emoção, competências sociais e emocionais, educação pré-escolar. 


\section{Introducción}

La educación emocional es en esencia un proceso educativo que busca el desarrollo de las dimensiones emocionales bajo el reconocimiento de las emociones así como el manejo y autorregulación de cada una de ellas, y que se propone como complemento a la educación cognitiva y tradicional que pone énfasis solo en el desarrollo de contenidos curriculares, y que busca lograr la construcción integral de la persona.

Dado que en la actualidad las demandas profesionales y laborales son cada vez más exigentes, muchas empresas, sobre todo las más competitivas, plantean como filosofía la importancia de poner mayor acento en las competencias socioemocionales, es decir en lo relacionado al saber ser.

Tal como lo sostiene Bisquerra (2003), desarrollar competencias tanto académicas como socioemocionales en nuestros niños y niñas es un hecho innegable, lo importante aquí es cómo trabajar en las escuelas y lograr la sinergia entre dichas competencias, sin dejar de lado los ritmos y procesos de aprendizaje propio de los niños para no caer en la yuxtaposición de competencias, donde muchas veces prima los conocimientos académicos, por encima de los socioemocionales.

Por ello, es importante considerar la enorme importancia que tiene la escuela en el desarrollo de la educación emocional, pues es prioritario lograr que los niños y las niñas tengan una educación de calidad basada en las competencias socioemocionales, como sostiene Bisquerra (2003).

Sobre este aspecto, Bisquerra (2003) plantea algunas dimensiones que pueden ser trabajadas desde la educación emocional como son: conciencia emocional, la inteligencia emocional, la regulación de las emociones así como el desarrollo de las competencias socioemocionales.

Con respecto a la Conciencia emocional, esta se refiere a la capacidad de tomar conciencia de las propias emociones y la de los demás, y que es muy importante en el proceso de socialización del ser humano.

La regulación de las emociones son para los niños un proceso que demanda tiempo y que le permite el desarrollo de su autonomía y seguridad ya que les permite 
manejar sus emociones e manera asertiva en diferentes contextos y situaciones donde se encuentre. Por ello la escuela cumple un rol importante al ofrecerle diferentes experiencias para que aprenda a manejar mejor sus emociones.

La inteligencia emocional es un aporte dado por Goleman (1997) quien desarrolla los conceptos sobre inteligencia intra e interpersonal y que dan por resultado que el individuo se relacione de manera positiva y tolerante primero consigo mismo y también con los demás.

Las competencias socioemocionales involucran la movilización de las diferentes emociones de los demás de manera empática comprendiendo lo que sienten los demás y que les permite relacionarse con otros de manera eficaz.

Por tal motivo debido a su relevancia en el campo educativo, la educación emocional toma un papel importante en el desarrollo pleno e integral del ser humano y que a través de este artículo permite la revisión de diferentes artículos que abordan la problemática y situación de esta temática en diferentes contextos educativos. De esta manera se analizan las propuestas educativas que pueden implementarse en las aulas para desarrollas el aspecto emocional de los niños del nivel preescolar.

En este sentido, a través de este artículo se presenta una revisión sistemática de estudios relacionados con la educación emocional y su implicancia en el desarrollo integral del niño del nivel preescolar teniendo en cuenta programas y estrategias acordes a las características de este grupo de estudiantes.

\section{Metodología}

Esta investigación constituye un análisis de revisión sistemática de diversos artículos académicos en un periodo desde el 2016 al 2020 a través de los buscadores de datos SCOPUS, EBSCO, SPRINGER, además de otros buscadores complementarios como Google académico. Las palabras clave utilizadas para esta búsqueda fueron conciencia emocional, inteligencia, emocional, regulación de las emociones y competencias sociales y emocionales. En inglés lo constituyeron las siguientes keywords: Emotional awareness, emotional intelligence, emotion regulation, social and emotional competencies. Se consideran para la búsqueda el uso de los operadores lógicos como "or" o "and” en caso que la búsqueda se realice en idioma inglés o sus equivalente en español como "o" o "y". 
Se incluyeron en la búsqueda todos los artículos de revistas indexadas y que están relacionados con la variable, el rango del grupo etáreo considerado, artículos de los años 2019 y 2020 y al menos dos o tres que se consideraron relevantes para el estudio del año 2016, 2017 y 2018; por otro lado se excluyeron aquellos artículos que no contaban con las características de la variable y con otras unidades de estudio y por contar con poca rigurosidad científica.

Se utilizó la metodología PRISMA para realizar el filtro y análisis de todos los artículos preseleccionados considerados 107 de los cuales quedaron 22 para el presente documento cuyos enfoque y tipos de estudio son cuantitativos, cualitativos y mixtos. Lo cual se evidencia en una matriz con los artículos incluidos en el presente estudio.

\section{Resultados}

Filtro de artículos mediante la Metodología PRISMA
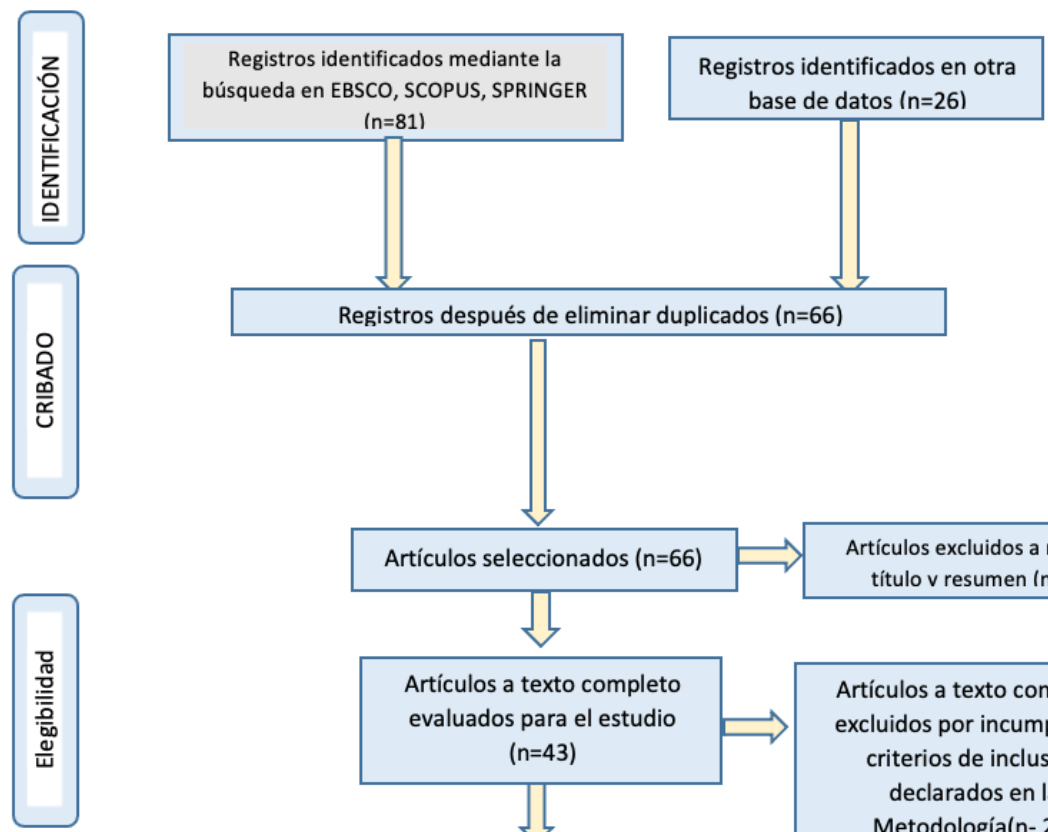

Registros después de eliminar duplicados $(n=66)$

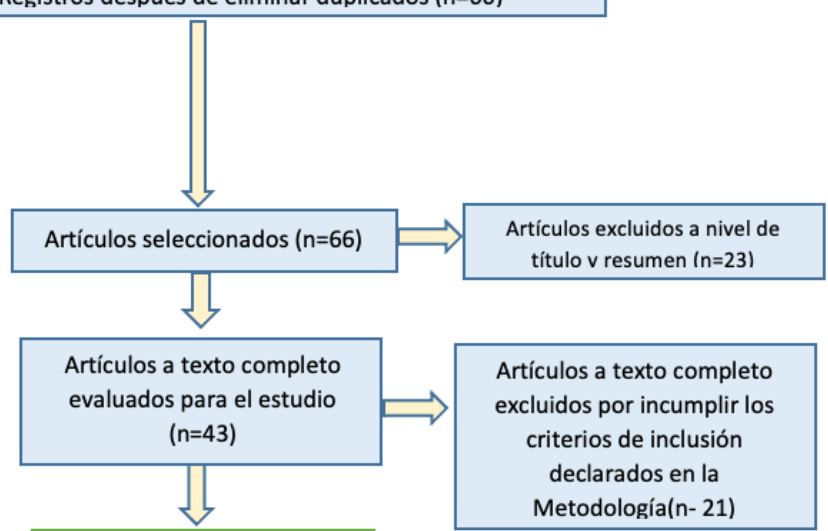

Artículos cuantitativos

incluidos en el estudio ( $\mathrm{n}-12)$
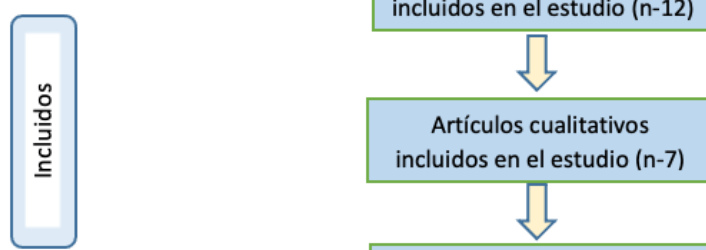

Artículos cualitativos incluidos en el estudio ( $n-7)$

Artículos mixtos incluidos en el estudio (n-3)

Fig.1 Diagrama adaptado de la Metodología Prisma para filtrar artículos (Moher et al., 2009) 


\begin{tabular}{|c|c|c|c|c|c|c|c|}
\hline \multirow[b]{2}{*}{$\begin{array}{l}\mathrm{N} \\
\mathrm{o} .\end{array}$} & \multirow[b]{2}{*}{ Autor/es } & \multicolumn{4}{|c|}{ Educación emocional en niños } & \multirow[b]{2}{*}{$\begin{array}{l}\text { Tipo de } \\
\text { estudio, } \\
\text { población y } \\
\text { muestra }\end{array}$} & \multirow[b]{2}{*}{$\begin{array}{l}\text { Instrumento, técnica o } \\
\text { metodología empleada }\end{array}$} \\
\hline & & $\begin{array}{l}\text { Conci } \\
\text { encia } \\
\text { emoci } \\
\text { onal }\end{array}$ & $\begin{array}{l}\text { Intelige } \\
\text { ncia } \\
\text { emocio } \\
\text { nal }\end{array}$ & $\begin{array}{l}\text { Regul } \\
\text { ación } \\
\text { de las } \\
\text { emoci } \\
\text { ones }\end{array}$ & $\begin{array}{l}\text { Compete } \\
\text { ncias } \\
\text { sociales } \\
\text { y } \\
\text { emocion } \\
\text { ales }\end{array}$ & & \\
\hline 1 & $\begin{array}{l}\text { (Calhoun et } \\
\text { al.,2020) }\end{array}$ & & & $\mathrm{X}$ & $\mathrm{X}$ & $\begin{array}{l}\text { Estudio } \\
\text { cuantitativo }\end{array}$ & $\begin{array}{l}\text { Baterías de pruebas } \\
\text { Sesiones de prueba } \\
\text { Escala de Competencia } \\
\text { social } \\
\text { Escala de actividad infantil }\end{array}$ \\
\hline 2 & $\begin{array}{l}\text { (Aguaded, } \\
2017)\end{array}$ & & $\mathrm{X}$ & $\mathrm{X}$ & $\mathrm{X}$ & $\begin{array}{l}\text { Estudio } \\
\text { cualitativo. }\end{array}$ & $\begin{array}{l}\text { Observación, diario de } \\
\text { clase, registro anecdótico. }\end{array}$ \\
\hline 3 & $\begin{array}{l}\text { (Fernández- } \\
\text { Hawrylak et al., } \\
\text { 2020) }\end{array}$ & $\mathrm{x}$ & & $\mathrm{x}$ & $\mathrm{x}$ & $\begin{array}{l}\text { Estudio } \\
\text { cuantitativo. }\end{array}$ & $\begin{array}{l}\text { Escala de Reconocimiento, } \\
\text { Regulación, Empatía y } \\
\text { Resolución de Problemas } \\
\text { (RRER). }\end{array}$ \\
\hline 4 & $\begin{array}{l}\text { (Damas et } \\
\text { al.,2020) }\end{array}$ & $\mathrm{x}$ & & $\mathrm{x}$ & $\mathrm{x}$ & $\begin{array}{l}\text { Estudio } \\
\text { cuantitativo, }\end{array}$ & $\begin{array}{l}\text { Cuestionario de Conciencia } \\
\text { Emocional (de Damas, } \\
\text { 2017) diseñado ad hoc. }\end{array}$ \\
\hline 5 & (Arce , 2020) & & $X$ & & $\mathrm{x}$ & $\begin{array}{l}\text { Estudio } \\
\text { cuantitativo. }\end{array}$ & $\begin{array}{l}\text { Pre test sobre inteligencia } \\
\text { emocional. Técnicas de } \\
\text { recolección de información } \\
\text { Observación: } \\
\text { Rúbrica de evaluación }\end{array}$ \\
\hline 6 & $\begin{array}{l}\text { (Alcoser- } \\
\text { Grijalva et al., } \\
\text { 2019) }\end{array}$ & $X$ & & $\mathrm{X}$ & $X$ & $\begin{array}{l}\text { Estudio de tipo } \\
\text { mixto. }\end{array}$ & $\begin{array}{l}\text { Observación, } \\
\text { entrevista, } \\
\text { Encuesta. }\end{array}$ \\
\hline 7 & $\begin{array}{l}\text { (Escorcia et } \\
\text { al.,2020) }\end{array}$ & $X$ & $\mathrm{X}$ & & $\mathrm{X}$ & $\begin{array}{l}\text { Estudio } \\
\text { cualitativo. }\end{array}$ & Cuestionario. \\
\hline 8 & $\begin{array}{l}\text { (García-Marín et } \\
\text { al.,2020) }\end{array}$ & & $\mathrm{X}$ & $\mathrm{X}$ & & $\begin{array}{l}\text { Estudio } \\
\text { cualitativo. }\end{array}$ & $\begin{array}{l}\text { Adaptación del test } \\
\text { PERVALEX (percibir y } \\
\text { expresar emociones de } \\
\text { forma precisa). }\end{array}$ \\
\hline 9 & $\begin{array}{l}\text { (Grimaldo et } \\
\text { al.,2020) }\end{array}$ & $X$ & & $\mathrm{X}$ & & $\begin{array}{l}\text { Estudio } \\
\text { cuantitativo. }\end{array}$ & $\begin{array}{l}\text { Técnicas participativas, } \\
\text { Lista de Chequeo } \\
\text { observacional. }\end{array}$ \\
\hline $\begin{array}{l}1 \\
0\end{array}$ & $\begin{array}{l}\text { (Cruz et al., } \\
2020)\end{array}$ & $X$ & & $X$ & & $\begin{array}{l}\text { Estudio de tipo } \\
\text { mixto. }\end{array}$ & $\begin{array}{l}\text { Técnicas de corte } \\
\text { cuantitativo (encuestas a } \\
\text { cuidadores o padres de } \\
\text { familia). } \\
\text { Técnicas de tipo cualitativo } \\
\text { (análisis de producciones de } \\
\text { los estudiantes, observación } \\
\text { participante y entrevista al } \\
\text { tutor o docente). } \\
\text { Cuestionario. }\end{array}$ \\
\hline
\end{tabular}




\begin{tabular}{|c|c|c|c|c|c|c|c|}
\hline $\begin{array}{l}1 \\
1\end{array}$ & $\begin{array}{l}\text { (Quesada et al., } \\
\text { 2019) }\end{array}$ & $X$ & & $X$ & $X$ & $\begin{array}{l}\text { Estudio } \\
\text { cuantitativo. }\end{array}$ & $\begin{array}{l}\text { Observación, Escala de } \\
\text { habilidades } \\
\text { socioemocionales para pre } \\
\text { escolares (EHSE), lista de } \\
\text { cotejo. }\end{array}$ \\
\hline $\begin{array}{l}1 \\
2\end{array}$ & $\begin{array}{l}\text { (Cabanzo et al., } \\
\text { 2020) }\end{array}$ & $X$ & & $\mathrm{X}$ & $X$ & $\begin{array}{l}\text { Estudio } \\
\text { cualitativo. }\end{array}$ & $\begin{array}{l}\text { Observación participante } \\
\text {,diarios de campo, la } \\
\text { encuesta con preguntas } \\
\text { mixtas, una entrevista } \\
\text { semiestructurada, } \\
\text { cuestionario, talleres } \\
\text { pedagógicos }\end{array}$ \\
\hline $\begin{array}{l}1 \\
3\end{array}$ & $\begin{array}{l}\text { (Tamayo et al., } \\
\text { 2020) }\end{array}$ & & $X$ & & $X$ & $\begin{array}{l}\text { Estudio } \\
\text { cualitativo }\end{array}$ & $\begin{array}{l}\text { Encuesta socio- } \\
\text { demográfica, cuestionario } \\
\text { emocional, talleres. }\end{array}$ \\
\hline $\begin{array}{l}1 \\
4\end{array}$ & $\begin{array}{l}\text { (Garaigordobil, } \\
\text { 2018) }\end{array}$ & $\mathrm{X}$ & & $\mathrm{X}$ & & $\begin{array}{l}\text { Estudio } \\
\text { cuantitativo. }\end{array}$ & $\begin{array}{l}\text { Programas de juego } \\
\text { cooperativo. } \\
\text { Instrumento de desarrollo } \\
\text { socioemocional y de } \\
\text { prevención de la conducta } \\
\text { violenta. }\end{array}$ \\
\hline $\begin{array}{l}1 \\
5\end{array}$ & $\begin{array}{l}\text { (Vilca et al., } \\
\text { 2019) }\end{array}$ & $\mathrm{X}$ & $X$ & $\mathrm{X}$ & $X$ & $\begin{array}{l}\text { Estudio } \\
\text { cuantitativo }\end{array}$ & $\begin{array}{l}\text { Escalas de lenguaje y } \\
\text { socioemocional de la Bailey } \\
\text { Scale of Infant and Toddler } \\
\text { Development III y una pauta } \\
\text { para codificar etiquetas } \\
\text { emocionales. }\end{array}$ \\
\hline $\begin{array}{l}1 \\
6\end{array}$ & $\begin{array}{l}\text { (Binti et al., } \\
\text { 2020) }\end{array}$ & & $X$ & & & $\begin{array}{l}\text { Estudio de tipo } \\
\text { mixto. }\end{array}$ & $\begin{array}{l}\text { Sesiones de observación } \\
\text { participante y las entrevistas } \\
\text { semi-estructuradas. }\end{array}$ \\
\hline $\begin{array}{l}1 \\
7\end{array}$ & (Joya, 2019) & $X$ & & $X$ & $X$ & $\begin{array}{l}\text { Estudio } \\
\text { cualitativo }\end{array}$ & $\begin{array}{l}\text { Observación, el diario } \\
\text { pedagógico, la rejilla de } \\
\text { evaluación y las fotografías. }\end{array}$ \\
\hline $\begin{array}{l}1 \\
8\end{array}$ & $\begin{array}{l}\text { (Sanchez et al., } \\
\text { 2019) }\end{array}$ & $\mathrm{X}$ & & $\mathrm{X}$ & $X$ & $\begin{array}{l}\text { Estudio } \\
\text { cualitativo. }\end{array}$ & $\begin{array}{l}\text { Cuestionario de Evaluación } \\
\text { de Competencias } \\
\text { Emocionales en Niños } \\
\text { Preescolares (CECEP). }\end{array}$ \\
\hline $\begin{array}{l}1 \\
9\end{array}$ & $\begin{array}{l}\text { (Heras et al., } \\
2016)\end{array}$ & $\mathrm{X}$ & & $\mathrm{X}$ & $X$ & $\begin{array}{l}\text { Estudio } \\
\text { cuantitativo. }\end{array}$ & $\begin{array}{l}\text { La Escala Reconocimiento, } \\
\text { Regulación, Empatía y } \\
\text { Resolución de Problemas } \\
\text { (RRER). }\end{array}$ \\
\hline $\begin{array}{l}2 \\
0\end{array}$ & $\begin{array}{l}\text { (Cepa et } \\
\text { al.,2016) }\end{array}$ & $\mathrm{X}$ & & $\mathrm{X}$ & $X$ & $\begin{array}{l}\text { Estudio } \\
\text { cuantitativo. }\end{array}$ & $\begin{array}{l}\text { Escala Reconocimiento, } \\
\text { Regulación, Empatía y } \\
\text { Resolución de Problemas } \\
\text { (RRER), }\end{array}$ \\
\hline $\begin{array}{l}2 \\
1\end{array}$ & (Muchiut, 2018) & $X$ & & $X$ & $X$ & $\begin{array}{l}\text { Estudio } \\
\text { cuantitativo. }\end{array}$ & $\begin{array}{l}\text { Escala de comportamiento } \\
\text { Preescolar y Jardín Infantil- } \\
\text { Subescala Problemas de } \\
\text { Conducta, Cuestionario de } \\
\text { Emociones. }\end{array}$ \\
\hline
\end{tabular}




\begin{tabular}{|c|c|c|c|c|c|c|}
\hline $\begin{array}{l}2 \\
2\end{array}$ & $\begin{array}{l}\text { (Arciniegas et } \\
\text { al., 2019) }\end{array}$ & $X$ & $X$ & $X$ & $\begin{array}{l}\text { Estudio } \\
\text { cualitativo. }\end{array}$ & $\begin{array}{l}\text { Diario de campo entrevistas } \\
\text { semiestructurada. Talleres } \\
\text { lúdicos e interactivos, } \\
\text { observación participante. }\end{array}$ \\
\hline
\end{tabular}

Tabla 1. Distribución de resultados por autores, categorías y metodología.

La Tabla 1 muestra la distribución de los resultados de la búsqueda por autor, años de edición, categorías y metodología. E1 72.7\% de los estudios corresponde a la subcategoría Conciencia emocional (de los 16 estudios 13 están interrelacionados con las demás subcategorías), el 31.8\% muestra la subcategoría Inteligencia emocional (De los 7 estudios ,6 están interrelacionados con las demás subcategorías).Un $81.8 \%$ se refiere a la subcategoría Regulación de las emociones (los 18 estudios están interrelacionados con las demás subcategorías).Un 77.2\% presenta a la subcategoría Competencias sociales y emocionales (Los 17 estudios de esta subcategoría están interrelacionados con otras subcategorías).

\section{Discusión}

Los resultados obtenidos nos indican que los conceptos "Conciencia emocional", "Inteligencia emocional", "Regulación de emociones" y "Competencias sociales y emocionales" son categorías referentes al tema, que nos permiten abordar la discusión planteada en la presente investigación.

Los primeros años de vida ejercen una fuerte influencia en el desarrollo de las habilidades que envuelven la conciencia emocional como parte del desarrollo integral estructurado en una determinada cultura y contexto. (Sánchez, 2019).

Teniendo en cuenta ello, la educación emocional cumple una función trascendental en el crecimiento y desarrollo pleno de la persona y por ende de la sociedad; de ahí su importancia en el sistema educativo. Al respecto, si bien es cierto que los sistemas educativos han estado orientados a promover conocimientos, estos se han enfocado principalmente en el desarrollo de la cognición, observándose que la parte emocional ha quedado relegada a un segundo plano, con poca presencia en los planes de estudio y la práctica pedagógica frecuente. 
Como apuntan los especialistas, la educación emocional es muy importante para el desarrollo de los niños y debe brindarse en las escuelas desde el nivel preescolar de manera sistemática. (García-Marín et al., 2020).

De esta manera, la educación emocional se va constituyendo como un proceso que busca el desarrollo del ámbito socioemocional de los estudiantes, formando una personalidad de manera integral, yendo de la mano con las competencias cognitivas que, como se ha señalado, por muchos años ha sido más impulsada en detrimento del aspecto emocional.

En ese sentido, muchas investigaciones apoyan la necesaria implementación de la educación emocional en las escuelas desde el nivel pre-escolar con la participación de miembros de la escuela y las familias, a través de programas que se impartan de manera sistemática, así como talleres que promuevan el desarrollo de sus competencias emocionales. (Heras et al., 2016).

Para lograr ello, se necesita una planificación de actividades muy significativas que tomen en cuenta los intereses y necesidades que atraviesan este grupo de estudiantes; y que coordinen un trabajo articulado entre la familia y la escuela, pues éstos espacios cimentan las primeras experiencias que los lleven a construir sus emociones, teniendo en cuenta la sana convivencia, lo cual lo llevará en un futuro a interactuar con los demás de manera eficiente y respetuosa.

Efectivamente, el proceso de construcción del desarrollo de las competencias sociales y emocionales se da a través de la escuela donde se producen diversos tipos de interacciones de los estudiantes. (Muchiut, 2018)

De ahí la influencia de la escuela en el campo de la formación y educación integral de la persona, que debe convertirse en una directriz que ayude a los estudiantes a desarrollarse emocionalmente fuera del ámbito familiar; sobre todo, porque en la escuela se producen muchas interacciones y vínculos cercanos a la que se dan en la sociedad en general.

De otro lado, considerando que en la actualidad las demandas profesionales y laborales son cada vez más exigentes, muchas empresas, sobre todo las más competitivas, plantean como filosofía la importancia de poner mayor acento en las competencias socioemocionales, es decir en lo relacionado al saber ser. 
Dichas competencias socioemocionales han empezado a ser vistas como un aspecto trascendental y decisivo para alcanzar la excelencia e influir en la vida de las personas, para trabajar mejor en equipo (Goleman, 2010) y alcanzar el éxito tanto en lo profesional como en lo personal, puesto que involucra poner en juego habilidades a nivel intrapersonal así como interpersonales, tal como sostiene Gardner $(2,016)$ en su Teoría sobre las Inteligencias Múltiples.

Es por eso que las emociones guardan una serie de características que las hacen únicas y especiales en los estudiantes, quienes desde edades tempranas deben aprender a manejarlas de acuerdo al contexto donde se desarrollen; los mecanismos de las emociones nos dan luces sobre su importancia en la adaptación del ser humano ante las circunstancias de la vida diaria. (Tamayo , 2020).

No obstante, como se ha señalado, desde hace muchos años las emociones han sido catalogadas como aspectos no claramente abordados en las escuelas, ya que se promueve en muchas de ellas solo el aspecto académico, descuidando el aspecto emocional de los estudiantes.

Desarrollar competencias tanto académicas como socioemocionales en nuestros niños y niñas es un hecho innegable, lo importante aquí es desarrollar estrategias para lograr la sinergia entre dichas competencias, sin dejar de lado los ritmos y procesos de aprendizaje propios de los niños, para no caer en la yuxtaposición de competencias, donde muchas veces priman los conocimientos académicos, por encima de los socioemocionales (Bisquerra, 2003).

Sin educación emocional en las instituciones educativas, los estudiantes presentan dificultades en su desenvolvimiento social, lo cual nos demuestra su importancia frente a la coyuntura actual de violencia y enfermedades psicológicas y de dependencia. (Aguaded, 2017).

Es indispensable contar con una clara politica de educación emocional en las escuales ya que permite educar a los estudiantes, de manera que aprendan a manejar sus emociones, haciéndolos cada vez más competentes de manera interpersonal como intrapersonal.

En muchos lugares del mundo, la aplicación de la educación emocional ha dado resultados positivos en sus estudiantes al llevarlos a desenvolverse de manera social a 
través de diferentes experiencias de aprendizaje que lo ayuden a tomar conciencia de sí mismos y reflexionar sobre la mejor manera de solucionar dificultades, de forma socialmente inteligente.

Así, la escuela se transforma en el espacio ideal para desarrollar la inteligencia emocional, lo cual permite a los estudiantes regular sus emociones de manera positiva a través de interacciones positivas que le ofrezcan la oportunidad de superar dificultades y solucionar problemas con inteligencia emocional. (Aguaded, 2017)

Vale añadir que las instituciones educativas siempre han sido consideradas como entidades fundamentales en el desarrollo del estudiante, como persona tanto en lo academico como en la parte emocional. La UNESCO a través del informe Delors (1996), resaltó la enorme preocupación por formar personas cada vez mejor preparadas para enfrentar los desafíos del nuevo milenio, para lo cual propuso cuatro pilares sobre los que se debe cimentar la educación para el siglo XXI : Aprender a conocer, aprender a hacer, aprender a convivir y aprender a ser. En ese sentido, es necesario poner sobre el tapete la importancia de las habilidades sociales y emocionales en los estudiantes para desarrollarse integralmente a lo largo de toda su vida y hacer frente a la incertidumbre de los tiempos actuales (Fragoso-Luzuriaga, 2015).

Por su parte, la "inteligencia emocional”, término que deviene de la psicología, sirve de base para el estudio de las emociones ya que su aporte en el campo educativo es muy importante. (Binti et al. , 2020).

Es así que los conceptos de inteligencia emocional llevan a un mejor entendimiento de la importancia que la educación emocional posee y que propende a través de los programas educativos que se llevan a cabo en las escuelas.

Esto se evidencia en el desarrollo de dos aspectos importantes presentes en la inteligencia emocional, como son sus áreas intra e interpersonal, lo cual significa las capacidades que la persona tiene de construirse así mismo personalmente, para luego expresar este crecimiento hacia los demás y crear vínculos positivos con ellos. (Arce,2020).

Efectivamente, los estudiantes del nivel preescolar evidencian una mejor interrelación a nivel personal y social al manejar sus emociones de manera asertiva luego de participar en programas de educación emocional; por lo que es fundamental 
generar espacios que conlleven a realizarse desde los primeros años y a lo largo de su vida. (Grimaldo et al, 2020).

Si bien el aspecto socioemocional está presente en cada una de las actividades educativas del nivel preescolar, muchas veces no ha sido sistematizado, observándose solo el aspecto cognitivo dejando de lado el aspecto emocional de los niños que forman parte de este nivel educativo.

Una implementación de la educación emocional promueve el desarrollo de la regulación de las emociones llevando al niño a su autoconomiento para poder entender lo que le pasa, para después entender a los demás siendo un proceso que se va dando en el tiempo. (Joya, 2019) Esto desarrolla en los niños la capacidad de saber conducirse de manera empática y asertiva con los demás, estableciendo mejores lazos de interacción social.

Esto es así, pues diversos estudios dan cuenta que es muy beneficioso desarrollar aspectos relacionados con la educación de las emociones desde los primeros años de vida, ya que les permitirá identificar emociones, describirlas y educarlas lo cual será de gran beneficio en su vida futura. (Cepa et al., 2016).

Después de todo, el fin último de la educación es lograr el desarrollo de la persona de manera integral y solo se logra a través de una formación no solo académica sino emocional ya que ambos aspectos se conjugan en la vida de todo ser humano.

Por ello, es importante considerar la enorme importancia que tiene la escuela en el desarrollo de las competencias socioemocionales, yendo más allá, pues es prioritario lograr que los niños y las niñas tengan una educación de calidad basada en las competencias socioemocionales, como sostiene Bisquerra (2003). Esto no desconoce que la familia es un agente que debe permitir que los niños afloren sus sentimientos y logren identificar las emociones, las vivan y aprendan a regularlas.

La regulación emocional se desarrolla a través de la educación emocional que permite a los niños modificar sus conductas de tal manera que pueda interrelacionar de manera asertiva y empática en el medio donde se desenvuelve. (Arciniegas et al. , 2019). 
Es por ello que esta regulación emocional debe estar presente en las diferentes experiencias de aprendizaje que promuevan la educación emocional .Tanto la educación como el aprendizaje son aspectos gravitantes en los niños y niñas, quienes por sus ritmos de desarrollo tienen la necesidad de adquirir no sólo conocimientos académicos, sino también desarrollar competencias socioemocionales para desarrollarse de manera armónica y gestionar de manera reflexiva y pertinente el mundo emocional que es preciso educar (Jiménez et al., 2009).

Justamente por estas funciones tan importantes, la educación emocional debe planificarse en todas las escuelas como parte del desarrollo personal de la persona en todos los niveles educativos y además en la sociedad en general. (Quesada et al., 2019). Actualmente el desarrollo de la persona se está observando de manera holística ya que no solo toma en cuenta la educación básica sino todas las demás etapas de la vida.

Es importante acotar que tradicionalmente se tenía la creencia que las emociones eran un impedimento para el desarrollo del ser humano, como del pensamiento, interrumpiendo el normal desarrollo de las personas, ya que no permitían el proceso del aprendizaje. Sin embargo, gracias a numerosas investigaciones y a los avances científicos, ahora se sabe que las competencias socioemocionales son un vehículo que permiten al ser humano pensar mejor y lograr un óptimo procesamiento de la información en la interacción consigo y para con los demás (Fragoso-Luzuriaga, 2015).

En muchas de nuestras escuelas se tiene por sentado que el desarrollo de las competencias socioemocionales de los niños y niñas se da de manera implícita en las actividades que se realizan (Cohen, 2003). Pero es importante detenernos y analizar si esto realmente se está llevando a cabo y qué consecuencias trae la ausencia de la educación emocional.

Según investigaciones realizadas por varios autores (Cerezo, 1996, 2001, 2006; Ortega y Mora-Merchán, 2000; Serrano e Iborra, 2005; Avilés y Monjas, 2005; Piñuel y Oñate, 2005), la violencia entre escolares muestran un incremento paulatino llegando a alcanzar un índice cercano al 40\%, lo cual pone en evidencia que el problema de la agresividad entre escolares afecta a un gran número de niños y adolescentes, tanto en su rendimiento académico como a su desarrollo personal ,creando un clima escolar 
aversivo y amenazante, que genera graves síntomas de ansiedad en los estudiantes agredidos (Cerezo, 2006; Piñuel y Oñate, 2005).

La educación emocional contribuiría a prevenir esas situaciones de violencia, pues incide en el crecimiento emocional de los estudiantes ya que al reconocerse en lo emocional también serán capaces de entender las emociones de los demás; por ello el papel de la escuela es no solo formarlos académicamente sino también en el plano de las emociones. (Cabanzo et al., 2020).

Por ello en cada escuela se tiene que adecuar la curricula al desarrollo emocional de acuerdo al contexto en que viven los estudiantes atendiendo a diversas problemáticas, como la violencia que se traducen en conductas disruptivas en los niños, que es muchas veces el reflejo del poco desarrollo de las emociones desde las primeras etapas de desarrollo.

La importancia de la educación emocional en las escuelas es cada vez más evidente por los enormes beneficios que brinda al desarrollo de la personalidad de los estudiantes creando un escudo protector a una serie de problemas futuros que puedan generar peligros en diversas situaciones de la vida debido a las competencias sociales y emocionales que posee el estudiante. (Garaigordobil, 2018)

El carácter preventivo de la educacion emocional se constituye en una gran oportunidad que ayuda a la formacion del estudiante y que se puede observar en las aulas de clase donde las diferentes personalidades se ponen en juego.

Queda así evidente que el papel de la escuela es crucial en la construcción de las competencias emocionales dependiendo del entorno sociocultural de los niños, contribuyendo así en la calidad de interacción social que se van desarrollando en este espacio social. (Vilca et al., 2019).

Las competencias emocionales se desarrollan en situaciones significativas a través de la movilización de las habilidades que el niño debe poner en juego, este proceso es gradual por ello cada actividad debe ser significativa en el contexto real donde viven los estudiantes.

Es muy importante señalar que este tipo de competencias se desarrollan a través de una metodología educativa que considera en su planificación y ejecución los 
conocimientos previos de los niños, es decir sus vivencias y experiencias personales intereses y necesidades, lo cual los lleva a socializarlas con los demás en su contexto en una cosmovisión en particular.

Una educación emocional bien planificada e implementada con la participación de docentes capacitados aporta enormes beneficios a los niños a través de programas que dirigidos hacia el desarrollo de las habilidades emocionales a la par con las académicas y con el apoyo de toda la comunidad educativa. (Muchiut, 2018)

El profesor que lleve a cabo los programas de educación emocional requiere continua preparación en todo sentido y con mayor razón en aspectos de las emociones buscando aliados estratégicos siendo la familia uno de los ejes primordiales para tal fin.

En la escuela el profesor ejerce una presencia importante, por lo cual debe tener en cuenta la libre expresión de las emociones de los estudiantes en un ambiente propicio de respeto y tolerancia dando paso a la regulación de todas las emociones que se presenten fortaleciendo, aquellas que permitan una sana convivencia en el aula y en otros espacios educativos.

Por ende, la educación emocional debe llevarse a cabo de manera estructurada en las escuelas y estar presente en la curricula y llevada a cabo por los propios docentes quienes deben asociar las experiencias de aprendizaje de acuerdo al contexto de los estudiantes. (Aguaded, 2017)

Muchos especialistas ponen en relieve la gran importancia que tiene la Educación emocional lo cual demanda que se realice de manera planificada y no de forma improvisada. Por otro lado, al implementar la educación emocional, la percepcion de los estudiantes es muy importante ya que observan la manera como se relacionan los adultos con ellos, como trasmiten sus valores,creencias y actitudes; muchas veces este aspecto pasa por alto y no se percibe en los contextos educativos.

La educación emocional desarrollada de manera sistemática en las escuelas promueve el desarrollo de un clima de sana convivencia en las aulas, siendo importante hacer uso de una metodología acorde a este tipo de educación considerando la participación de padres y profesores. Por ello como principal punto a bordar es la preparación de los maestros en lo que se refiere a educación emocional. (AlcoserGrijalva et al. , 2019). 
Es muy importante que en las aulas siempre exista un buen clima en relación a adecuadas relaciones interpersonales entre el docente y los alumnos y entre los propios alumnos y se generen los aprendizajes de manera armoniosa. Uno de los indicadores más evidentes en cuanto al manejo de las emociones lo constituye la convivencia en el aula; porque es en el aula donde debe promoverse paz y diálogo respetuoso, los estudiantes se sentirán valorados y tranquilos para aprender ya que esta seguridad deviene de la buena gestión de las emociones de parte del maestro y entre los mismos estudiantes.

La forma como se da en las escuelas debe ser realizada en coordinacion con toda la comunidad educativa para que se vea reflejada en cada una de las actuaciones diarias de los estudiantes desde niveles tempranos como es el nivel preescolar. Aunque también es necesario desarrollarla en las diferentes etapas de la vida es decir en el ciclo vital de las personas.

Como se puede ver, un aspecto fundamental a considerar en el proceso de implementación de la educación emocional en las escuelas es la participación de los profesores ya que a través de las experiencias planificadas promueven aprendizajes significativos en torno a temas de las emociones de sus estudiantes contribuyendo al desarrollo de su mundo emocional. (Cruz et al., 2020).

Es por ello que en toda escuela dichos programas deben estar presentes en sus documentos de gestión sea PEI, PAT para darle un sentido institucional teniendo en cuenta el currículo escolar que promueve esta clase de experiencias educativas desde los primeros niveles de la educación. Es muy importante señalar que la educación emocional tiene el propósito desarrollar las competencias positivas para la interacción social y que en alguna manera servirá como medida preventiva ante tantos casos de violencia y problemas de conductas disruptivas.

La planificación de programas de educación emocional basados en la psicomotricidad beneficia positivamente la construcción de la inteligencia emocional en los niños preescolares ya que es una de las estrategias que despierta el interés de este grupo de niños. (Arce ,2020).

La psicomotricidad es una excelente estrategia como parte de la educación emocional para el desarrollo de las emociones ya que sintoniza con los intereses propios 
de los niños y que los ayuda a expresar todo sus emociones. Las actividades educativas de este tipo despiertan el interés de los estudiantes y son en gran medida muy útiles a la hora de pedir a los niños la expresión de sus emociones en diferentes situaciones.

Los Programas de educación emocional repercuten en el desarrollo personal y social de los niños de manera favorable, promoviendo interacciones positivas entre los mismos niños y profesores lo cual conlleva al desarrollo de sus habilidades sociales, emocionales y cognitivas.(Calhoun et al.,2020).

El campo de la educación emocional está teniendo gran relevancia en muchos sistemas educativos del mundo por lo que surgen diversas propuestas o programas educativos que sientan las bases de este tipo de educación en las escuelas para ser abordada de manera sistemática a fin de ayudar a los estudiantes a desarrollar plenamente su personalidad.

Dada la complejidad que tiene la competencia emocional se debe llevar a cabo considerando sus distintas dimensiones en los programas de educación emocional que se lleven a cabo en las escuelas. (Escorcia et al., 2020).

Las escuelas deben considerar los distintos aspectos que involucran las competencias emocionales lo cual deben ser estudiados y analizados por los docentes que llevaran a cabo esta tarea en las escuelas.

En muchos lugares del mundo como España las propuestas de educación emocional son muy escasas sobre todas las dirigidas hacia los 2 a 3 primeros años de vida que conlleven al desarrollo de la conciencia emocional y competencias emocionales; por otro lado si se han presentado programa de educación emocional en niños de nivel preescolar de 3 a 5 años. (Damas et al., 2020).

De allí que surge como propuesta innovadora en muchos sistemas educativos siendo indispensable tomar en consideración la importancia de la educación emocional de manera organizada a fin de coadyuvar con el desarrollo de la personalidad del niño de manera holística, basada en el autoconocimiento de sus propias emociones para que lo lleve a interactuar con otros con mayor inteligencia y siendo emocionalmente más competente. 
En otras palabras para el desarrollo del aspecto emocional es necesario realizar actividades que permitan la libre expresión de los niños, que les permita reflexionar de manera personal para luego compartir en actividades grupales e intercambiar sus percepciones y puntos de vista y estos espacios se favorecen a través de programas de educación emocional bien planificados.

\section{Conclusiones}

La educación emocional es uno de los grandes pendientes para desarrollar integralmente la formación de nuestros niños y niñas desde las escuelas. Su importancia se ha visto respaldada en diversas investigaciones y en experiencias de otros países.

Reflexionar sobre la inclusión de la educación emocional en nuestras escuelas de una manera más visible, integral y programática, dado que no se encuentra consolidado. Para ofrecer una educación de calidad el perfil ideal del educador para este nuevo siglo, de acuerdo a la política del Marco del Buen Desempeño Docente, aprobado por el Ministerio de Educación, señala que como docentes tenemos que dar prioridad a la formación y el relacionamiento socioemocional con nuestros estudiantes; de ahí, el papel fundamental de la escuela en este ámbito.

De este modo, los resultados obtenidos en esta investigación pueden ser muy útiles para otras investigaciones sobre el tema ya que luego del análisis sobre las variables permite seguir investigando y enriqueciendo el conocimiento acerca de la dimensión emocional de los niños del nivel preescolar y que sería un aporte importante en el campo educativo como innovación educativa y como parte de medidas preventivas de conductas disruptivas o violentas en las aulas.

\section{Referencias}

Aguaded, M. (2017). Estrategias para potenciar la inteligencia emocional en educación infantil: aplicación del modelo de Mayer y Salovey / Strategies to enhance emotional intelligence in early childhood education: application of Mayer and Salovey. Tendencias Pedagógicas, 30(2017), 175-190. https://doi.org/10.15366/tp2017.30.010 
Alcoser-Grijalva, R., B., ;, Moreno-Ronquillo, B., ;, \& León-García, M. (2019). La educación emocional y su incidencia en el aprendizaje de la convivencia en inicial 2. Revista Ciencia UNEMI, 12.

Arce, K. (2020). Modelo educativo "juego, siento, soy" e inteligencia emocional en niños de educación inicial Educational model "I play, I feel, I am" and emotional intelligence in early education children. SCIÉNDO, 23(1), 59-64. https://doi.org/10.17268/sciendo.2020.009

Arciniegas, D., Meneses, B., Ribero, L., Rojas, L. (2019). Educación empocional para dar respuesta a los comportaminetos de niños entre tres a siete años de una institución privada. $U N A B, 53(9), 1689-1699$.

Binti, A., Izzamuddin, M., Binti M., Azlina, Khan, A., Ghana, G. (2020). Ei rubrics for preschool children. Universal Journal of Educational Research, 8(11), 1-5. https://doi.org/10.13189/ujer.2020.082301

Bisquerra, R., Pérez, N. (2003).Las competencias emocionales. Universidad de Barcelona -España.

Cabanzo, T., Gonzáles, J. (2020). ¡Mi vida es emocionante!: Propuesta pedagógica desde la lúdica y el apoyo de la familia para favorecer el desarrollo socio-afectivo en niños y niñas de 2 años de edad. CAU Bucaramanga.

Calhoun, B., Williams, J., Greenberg, M., Domitrovich, S., Russell, M., Fishbein, D. (2020). Social Emotional Learning Program Boosts Early Social and Behavioral Skills in Low-Income Urban Children | Enhanced Reader. Frontiers in Psychology. chrome-extension://dagcmkpagjlhakfdhnbomgmjdpkdklff/enhancedreader.html?pdf=https $\% 3 \mathrm{~A} \% 2 \mathrm{~F} \% 2 \mathrm{Fbrxt}$.mendeley.com\%2Fdocument $\% 2 \mathrm{Fconten}$ t\%2Ff367470d-39cd-3e34-9127-0af1505a8755

Cepa, A., Heras, D., Lara, F. (2016). Desarrollo Emocional: Evaluación De Las Competencias Emocionales En La Infancia. International Journal of Developmental and Educational Psychology. Revista INFAD de Psicología., 1(1), 75. https://doi.org/10.17060/ijodaep.2016.n1.v1.212 
Cerezo, F. (2006). Violencia y victimización entre escolares. El bullying: estrategias de identificación y elementos para la intervención a través del Test Bull-S. Revista Electrónica de Investigación Psicoeducativa, 9(4).

Cohen, J. (2003).La inteligencia emocional en el aula. Editorial Troquel, Argentina.

Cruz, P., D., \& Borjas, M., López-Calvo, M. (2020). Ludoevaluación de la emoción del miedo en educación infantil. Revista Latinoamericana de Ciencias Sociales,Niñez y Juventud, 19(1), 1-21.

Damas, M. y Gomariz, M. (2020). La verbalización de las emociones en Educación Infantil. Evaluación de un Programa de Conciencia Emocional. Estudios Sobre Educación, Vol 38, Pp 279-302 (2020). https://doi.org/10.15581/004.38.279-302

Delors, J. (1996.): “Los cuatro pilares de la educación” en La educación encierra un tesoro. Informe a la UNESCO de la Comisión internacional sobre la educación para el siglo XXI, Madrid, España: Santillana/UNESCO.

Escorcia, C., Díaz, P. (2020). Competencia emocional en atención temprana. Educación, 43, 1-16.

Fernández-Hawrylak M, Heras-Sevilla D, C. S. A. (2020). Evaluation of the Effectiveness of the Emotional Education Program EMO-ACTION within a Preschool Setting. Estudios Sobre Educación, 39, 87-107. https://doi.org/10.15581/004.39.87-107

Fragoso-Luzuriaga, R. (2015). “Inteligencia emocional y competencias emocionales en educación superior, ¿un mismo concepto?”, en Revista Iberoamericana de Educación Superior (RIES), México, UNAM-IISUE/Universia, vol. VI, núm. 16, pp. 110-125. https://ries.universia.net/article/view/1085/inteligencia-emocionalcompetenciasemocionales-educacion-superior-un-concepto.

Garaigordobil, M. (2018). Educacion emocional en la infancia y la adolescencia. Participación Educativa., 5(8), 107-127.

García-Marín, P., Casabella, M. (2020). Motriemoción : reconocimiento de emociones en preescolares a través de la motricidad .Motriemotion : emotion recognition 
through motor. Trances:Revista de Transmisión Del Conocimiento Educativo y de La Salud., 12(4), 433-461.

Gardner, H. (2016).Estructuras de la mente. Teorías de las Inteligencias Múltiples. Ciudad de México, México.

Goleman, D. (2010).La práctica de la Inteligencia Emocional. Barcelona: Kairós.

Grimaldo, M., y Merino-Soto, C. (2020). Efectos de un Programa de Intervención sobre las habilidades emocionales en niños preescolares. REOP - Revista Española de Orientación y Psicopedagogía, 31(1), 62. https://doi.org/10.5944/reop.vol.31.num.1.2020.27290

Heras, D., Cepa, A., y Lara, F. (2016). Desarrollo Emocional en la Infancia.un estudio sobre las competencias emocionales de niños y niñas. Infad-Revista de Psicología, 1(0214-9877). https://www.redalyc.org/pdf/3498/349851776008.pdf

Jiménez, M., López-Zafra, E. (2009) .Inteligencia emocional y rendimiento escolar: estado actual de la cuestión. Revista Latinoamericana de Psicología, vol. 41, núm. 1, pp. 69-79 Fundación Universitaria Konrad Lorenz Bogotá, Colombia

Joya, D. (2019). Estrategias lúdicas para fomentar el manejo de las emociones. Universidad de Bucaramanga.

Moher D, Cook DJ, Eastwood S, Olkin I, Rennie D, Stroup DF. Improving the quality of reports of meta-analyses of randomised controlled trials: the QUOROM statement. (2009). Quality of Reporting of Meta-analyses. Lancet. 1999; 354: 1896-900.

Muchiut, Á. (2018). Educación emocional, una deuda pendiente en nuestros salones de preescolar. Revista de Educación, 1, 137-152.

Piñuel, I. y Oñate, A. (2005). Informe Cisneros VII: violencia y acoso escolar en alumnos de primaria, ESO y Bachiller (Informe preliminar). Instituto de Innovación Educativa y Desarrollo Directivo. (http://www.acosoescolar.com)

Quesada, S., Otiniano, J., Poma, M. (2019). Influencia de la educación emocional en el desarrollo integral de los niños. Helios, 38(1), 10. 
Sanchez, A., Fragoso, R. (2019). Evaluación de competencias emocionales en preescolar. Revista RELEP, 1, 29-36.

Tamayo Zuluaga, D. M., López, L. T., Muñoz, M. F., Sierra, S. S., \& Palacios, Y. A. (2020). Emociones constructoras de paz desde los niños y las niñas del grado transición: representaciones desde su experienciaEmociones constructoras de paz desde los niños y niñas del grado Transición TT - Emociones constructoras de paz desde los niños y las niñas. Zona Próxima, 32. https://bbibliograficas.ucc.edu.co/docview/2348752919?accountid=44394

Vilca, D., y Farkas, C. (2019). Language and use of emotional labels: Their relationship with socioemotional development in 30-month-old children attending daycare. Psykhe, 28(2), 1-14. https://doi.org/10.7764/psykhe.28.2.1114 